Article

\title{
Cosmological Effects of Quantum Vacuum Condensates
}

\author{
Antonio Capolupo \\ Dipartimento di Fisica E.R.Caianiello and INFN Gruppo Collegato di Salerno, Universitá di Salerno, \\ 84084 Fisciano (SA), Italy; capolupo@sa.infn.it
}

Received: 22 October 2017; Accepted: 6 December 2017; Published: 12 December 2017

\begin{abstract}
In quantum field theory, many phenomena are characterized by a condensed structure of their vacua. Such a structure is responsible of a non trivial vacuum energy. Here we analyze disparate systems and we show that the thermal vacuum state for hot plasmas, the vacuum for boson field in curved space and the vacuum for mixed neutrinos have the state equation of dark matter, $w=0$, and values of the energy density which are in agreement with the one estimated for dark matter. Moreover, we show that the vacuum of axions mixed with photons has the state equation of the cosmological constant $w=-1$ and a value of the energy density compatible with the one of dark energy.
\end{abstract}

Keywords: Dark energy; dark matter; quantum vacuum

\section{Introduction}

Many independent observations, strengthened by the recent measurements of the CMBR spectrum, due to the WMAP experiment, and by the extension of the SNeIa Hubble diagram to redshifts higher than 1 [1-5], show that the geometry of the universe is spatially flat and the universe is in a phase of accelerated expansion. Such an expansion is thought to be due to a non-clustered fluid with negative pressure, called dark energy. Many models trying to explain this phenomenon have been proposed [6-32].

On the other hand, the discrepancy between the observed rotation curve of spiral galaxies, which remains flat also at large distance from the centre of the galaxy, and the Kepler's second law has been explained by postulating the existence of a hypothetical type of matter, called dark matter, distinct from baryonic matter, neutrinos and dark energy [8,33]. Such a form of matter has a vanishing pressure and then a state equation $w=p / \rho=0$. It has never been directly observed, however, many candidates have been proposed to try to solve the dark matter problem [34-39]. The explanation of dark components of the universe represents still a very big challenge of the fundamental physics.

Motivated by these facts, we have devoted our attention to the study of dark energy and dark matter. In this paper, we report the recent results [40] showing that the vacuum condensate structure of many phenomena, such as particle mixing and fields in curved space [41-54], generates non trivial contributions to the energy of the universe [40], which can represent new components of dark energy and dark matter. In particular, dark matter components can be provided by the thermal vacuum of the hot plasma present at the center of a galaxy cluster, by the vacuum of fields in curved space [55] and by the flavor neutrino vacuum. Such condensates indeed have state equation $w=0$ and values of the energy density of order of $\rho \sim 10^{-47} \mathrm{GeV}^{4}$ which are in agreement with $w$ and $\rho$ of dark matter, respectively. Moreover, a dark energy contribution is generated by the vacuum condensate characterizing the axion-photon mixing. Such a condensate has a behavior completely different with respect to the ones of the condensates mentioned above. Indeed its state equation is $w=-1$, which is the one of the cosmological constant and the value of the vacuum energy density is compatible with 
the one inferred from the observations for dark energy, $10^{-47} \mathrm{GeV}^{4}$ [40]. The results here presented establish a link between cosmology, particle physics and quantum field theory.

In the following, we introduce the Bogoliubov transformations in quantum field theory (QFT), which describe all the phenomena characterized by vacuum condensates. We present the energy density, the pressure and the state equation of the condensate induced by a generic Bogoliubov transformation. Then, we study the particular cases of thermal states, of fields in curved space and of particle mixing and we derive their state equations and their contributions to the dark sector of the universe.

\section{Bogoliubov Transformation and Vacuum Condensate}

Let us consider a Bogoliubov transformation for bosons (similar transformation holds for fermions) in the framework of QFT, $a_{\mathbf{k}}(\xi, t)=U_{\mathbf{k}}^{B} a_{\mathbf{k}}(t)-V_{-\mathbf{k}}^{B} a_{-\mathbf{k}}^{\dagger}(t)$. Here $a_{\mathbf{k}}$, are annihilators of the vacuum $|0\rangle, \xi$ is a parameter depending on the system analyzed and the coefficients $U_{\mathbf{k}}^{B}, V_{\mathbf{k}}^{B}$, satisfy the relation $\left|U_{\mathbf{k}}^{B}\right|^{2}-\left|V_{\mathbf{k}}^{B}\right|^{2}=1$.

By introducing the generator $J(\xi, t)$ of the Bogoliubov transformation, one can rewrite such a relation as $a_{\mathbf{k}}(\xi, t)=J^{-1}(\xi, t) a_{\mathbf{k}}(t) J(\xi, t)$, where $J(\xi, t)$ has the property, $J^{-1}(\xi)=J(-\xi)$. The vacuum annihilated by $a_{\mathbf{k}}(\xi, t)$, denoted with $|0(\xi, t)\rangle$, is related to the original vacua $|0\rangle$ by $|0(\xi, t)\rangle=J^{-1}(\xi, t)|0\rangle$. Such a relation which is a unitary operation in quantum mechanics, in QFT is not a unitary transformation any more, since $\mathbf{k}$ assumes a continuous infinity of values. In this case, $|0(\xi, t)\rangle$ and $|0\rangle$ are unitarily inequivalent and the physical vacuum of the system is $|0(\xi, t)\rangle[47,48]$. Notice that $|0(\xi, t)\rangle$ has a condensate structure. Indeed the average value of the number operator, $N_{\mathbf{k}}=a_{\mathbf{k}}^{\dagger} a_{\mathbf{k}}$, on $|0(\xi, t)\rangle$ is different from zero and it is given by $\left\langle 0(\xi, t)\left|a_{\mathbf{k}}^{\dagger} a_{\mathbf{k}}\right| 0(\xi, t)\right\rangle=\left|V_{\mathbf{k}}\right|^{2}$. This fact induces a non-zero expectation value of the free energy momentum tensor for real scalar fields and for Majorana fields on $|0(\xi, t)\rangle_{\lambda}$. We denote such terms as,

$$
\Xi_{\mu v}^{\lambda}(x) \equiv_{\lambda}\left\langle 0(\xi, t)\left|: T_{\mu v}^{\lambda}(x):\right| 0(\xi, t)\right\rangle_{\lambda},
$$

where $\lambda=B, F$ (with $B=$ bosons, $F=$ fermions) and the symbol $: \ldots$ :, indicates the normal ordering with respect to the original vacuum $|0\rangle_{\lambda}$.

\section{Energy Density, Pressure and State Equation of Vacuum Condensate}

The energy momentum tensor of the condensate $\Xi_{\mu v}^{\lambda}(x)$ behaves as a perfect fluid. Indeed, its off-diagonal components are zero, i.e., $\Xi_{i, j}^{\lambda}(x)=0$, for $i \neq j$. Then, one can define the energy density and pressure of the condensate as $\rho^{\lambda}=\Xi_{00}^{\lambda}(x)$, and $p^{\lambda}=\Xi_{j j}^{\lambda}(x)$, respectively. By computing the expectation values of Equation $(1)$ for the $(0,0)$ and $(j, j)$ components, one finds the explicit expressions of the energy density and of the pressure. For bosons, in the case of the isotropy of the momenta, $\rho_{B}, p_{B}$ and the state equation $w_{B}=p_{B} / \rho_{B}$ are [40]

$$
\begin{aligned}
\rho_{B} & =\frac{1}{2 \pi^{2}} \int_{0}^{\infty} d k k^{2} \omega_{k}\left|V_{k}^{B}\right|^{2}, \\
p_{B} & =\frac{1}{6 \pi^{2}} \int_{0}^{\infty} d k k^{2}\left[\frac{k^{2}}{\omega_{k}}\left|V_{k}^{B}\right|^{2}-\left(\frac{k^{2}}{\omega_{k}}+\frac{3 m^{2}}{2 \omega_{k}}\right)\left|U_{k}^{B}\right|\left|V_{k}^{B}\right| \cos \left(\omega_{k} t\right)\right], \\
w_{B} & =\frac{1}{3} \frac{\int d^{3} \mathbf{k} \frac{k^{2}}{\omega_{k}}\left|V_{k}^{B}\right|^{2}}{d^{3} \mathbf{k} \omega_{k}\left|V_{k}^{B}\right|^{2}}-\frac{1}{3} \frac{\int d^{3} \mathbf{k}\left(\frac{k^{2}}{\omega_{k}}+\frac{3 m^{2}}{2 \omega_{k}}\right) U_{k}^{B} V_{k}^{B} \cos \left(\omega_{k} t\right)}{\int d^{3} \mathbf{k} \omega_{k}\left|V_{k}^{B}\right|^{2}}
\end{aligned}
$$


respectively. On the other hand, for fermions one has [40]

$$
\begin{aligned}
\rho_{F} & =\frac{1}{\pi^{2}} \int_{0}^{\infty} d k k^{2} \omega_{k}\left|V_{k}^{F}\right|^{2}, \\
p_{F} & =\frac{1}{3 \pi^{2}} \int_{0}^{\infty} d k \frac{k^{4}}{\omega_{k}}\left|V_{k}^{F}\right|^{2}, \\
w_{F} & =\frac{1}{3} \frac{\int d^{3} \mathbf{k} \frac{k^{2}}{\omega_{k}}\left|V_{k}^{F}\right|^{2}}{\int d^{3} \mathbf{k} \omega_{k}\left|V_{k}^{F}\right|^{2}} .
\end{aligned}
$$

Such equations hold for many systems described by Bogoliubov transformations. Using the explicit form of $U_{k}$ and $V_{k}$, we analyze the cases of thermal states and of fields in curved space. Then, by modifying Equations (2)-(7), we study the particle mixing phenomenon which, at the level of annihilators, is represented by a rotation and a Bogoliubov transformation [52-54].

\section{Thermal States and Vacuum Contributions}

In the framework of the Thermo Field Dynamics (TFD) [46-48], a Bogoliubov transformation is used to introduce the thermal vacuum state $|0(\xi(\beta))\rangle_{\lambda}$, which is the physical vacuum of a system at non-zero temperature. Here $\beta \equiv 1 /\left(k_{B} T\right), k_{B}$ is the Boltzmann constant and $\lambda=B, F$. The state $|0(\xi(\beta))\rangle_{\lambda}$ is defined in order that the thermal statistical average $\mathcal{N}_{\chi_{\mathbf{k}}}(\xi)$ is given by $\mathcal{N}_{\chi_{\mathbf{k}}}(\xi)={ }_{\lambda}\left\langle 0(\xi(\beta))\left|\chi_{\mathbf{k}}^{\dagger} \chi_{\mathbf{k}}\right| 0(\xi(\beta))\right\rangle_{\lambda}$, where $\chi$ is the annihilator $(\chi=a$ for bosons and $\alpha$ for fermions) [47].

The Bogoliubov coefficients are given by $U_{\mathbf{k}}^{T}=\sqrt{\frac{e^{\beta \omega_{\mathbf{k}}}}{e^{\beta \omega_{\mathbf{k}} \pm 1}}}$ and $V_{\mathbf{k}}^{T}=\sqrt{\frac{1}{e^{\beta \omega_{\mathbf{k}} \pm 1}}}$, with - for bosons and + for fermions, and $\omega_{\mathbf{k}}=\sqrt{k^{2}+m^{2}}$. Then, by using Equations (2), (3), (5) and (6) one finds the following contributions of the thermal vacuum states to the energy and pressure. For temperatures of order of the cosmic microwave radiation, i.e., $T=2.72 \mathrm{~K}$, one find that photons and particles with masses of order of $\left(10^{-3}-10^{-4}\right) \mathrm{eV}$ contribute to the energy radiation with $\rho \sim 10^{-51} \mathrm{GeV}^{4}$ and state equations, $w=1 / 3$ [56]. On the other hand, non-relativistic particles give negligible contributions. Moreover, the thermal vacuum of the hot plasma filling the center of galaxy clusters, which has temperatures of order of $(10 \div 100) \times 10^{6} \mathrm{~K}$, has an energy density of $\left(10^{-48}-10^{-47}\right) \mathrm{GeV}^{4}$, pressure $p \sim 0$ and then, state equation $w \sim 0$. Such values of $\rho$ and $w$ are in agreement with the ones of dark matter. Therefore, the thermal vacuum state of hot plasma behaves as a dark matter component.

Unruh and of the Hawking effects can be also represented by thermal states. However, the temperatures for such phenomenon are very low and their contributions to the vacuum energy are negligible [56].

\section{Vacuum Contribution of Fields in Curved Background}

Let us now study the contribution to the energy density and pressure of a field in curved space [51]. The expression of such a contribution and its numerical value depends on the metric analyzed and on the regularization adopted. Here, we consider the spatially flat Friedmann Robertson-Walker metric $d s^{2}=d t^{2}-a^{2}(t) d \mathbf{x}^{2}=a^{2}(\eta)\left(d \eta^{2}-d \mathbf{x}^{2}\right)$, where $a$ is the scale factor, $t$ is the comoving time, $\eta$ is the conformal time, $\eta(t)=\int_{t_{0}}^{t} \frac{d t}{a(t)}$, with $t_{0}$ arbitrary constant. Moreover, we consider the cut-off regularization. The energy density and pressure of a boson field are $[57,58]$

$$
\begin{aligned}
& \rho_{\text {curv }}=\frac{2 \pi}{a^{2}} \int_{0}^{K} d k k^{2}\left(\left|\phi_{k}^{\prime}\right|^{2}+k^{2}\left|\phi_{k}\right|^{2}+m^{2}\left|\phi_{k}\right|^{2}\right), \\
& p_{\text {curv }}=\frac{2 \pi}{a^{2}} \int_{0}^{K} d k k^{2}\left(\left|\phi_{k}^{\prime}\right|^{2}-\frac{k^{2}}{3}\left|\phi_{k}\right|^{2}-m^{2}\left|\phi_{k}\right|^{2}\right) .
\end{aligned}
$$


where $\phi_{k}$ are mode functions, $\phi_{k}^{\prime}$ are the derivative of $\phi_{k}$ with respect to $\eta$ and $K$ is the cut-off on the momenta. By following Referefce [55], we use a cutoff on the momenta much smaller than the comoving mass of the field, $K \ll m a$ and we set $m \gg H$. With this choice of $K$, one has $\rho_{\text {curv }}=\frac{m K^{3}}{12 \pi^{2} a^{3}}$, $p_{\text {curv }} \sim 0$, and the state equation is the one of dark matter, $w_{\text {curv }} \simeq 0$. Notice that the energy density is much smaller than $\frac{m^{4}}{12 \pi^{2}}$, thus, light particles can produce contributions compatible with the estimated value of dark matter.

\section{Vacuum Contributions of Particle Mixing}

Let us now consider the mixing between two fields

$$
\begin{aligned}
& \varphi_{1}(\theta, x)=\varphi_{1}(x) \cos (\theta)+\varphi_{2}(x) \sin (\theta), \\
& \varphi_{2}(\theta, x)=-\varphi_{1}(x) \sin (\theta)+\varphi_{2}(x) \cos (\theta),
\end{aligned}
$$

where $\theta$ is the mixing angle, $\varphi_{i}(x)$, with $i=1,2$ are the free fields and $\varphi_{i}(\theta, x)$ are the mixed fields, which represent fermions as neutrinos and quarks, or bosons as axions, kaons, $B^{0}, D^{0}$, and $\eta-\eta^{\prime}$ systems.

By means of the generator $J(\theta, t)$, one can express the transformations (10) as $\varphi_{i}(\theta, x) \equiv$ $J^{-1}(\theta, t) \varphi_{i}(x) J(\theta, t)$. Moreover, one can define the mixed annihilators as $\chi_{\mathbf{k}, i}^{r}(\theta, t) \equiv$ $J^{-1}(\theta, t) \chi_{\mathbf{k}, i}^{r}(t) J(\theta, t)$, (with $\chi_{\mathbf{k}, i}^{r}=a_{\mathbf{k}, i}, \alpha_{\mathbf{k}, i}^{r}$, for bosons and fermion), and one can derive the physical vacuum as $|0(\theta, t)\rangle \equiv J^{-1}(\theta, t)|0\rangle_{1,2}$, where $|0\rangle_{1,2}$ is the vacuum annihilated by $\chi_{\mathbf{k}, i}^{r}[52,53] .|0(\theta, t)\rangle$ has a condensation density given by ${ }_{\lambda}\left\langle 0(\theta, t)\left|\chi_{\mathbf{k}, i}^{r+} \chi_{\mathbf{k}, i}^{r}\right| 0(\theta, t)\right\rangle_{\lambda}=\sin ^{2} \theta\left|Y_{\mathbf{k}}^{\lambda}\right|^{2}$, where $\lambda=B, F, i=1,2$ and the reference frame $\mathbf{k}=(0,0,|\mathbf{k}|)$ has been adopted [59-64]. The Bogoliubov coefficients for mixed bosons are, $\left|\mathrm{Y}_{\mathbf{k}}^{B}\right|=\frac{1}{2}\left(\sqrt{\frac{\Omega_{k, 1}}{\Omega_{k, 2}}}-\sqrt{\frac{\Omega_{k, 2}}{\Omega_{k, 1}}}\right)$ and $\Sigma_{\mathbf{k}}^{B}=\sqrt{1+\left|\mathrm{Y}_{\mathbf{k}}^{B}\right|^{2}}$, and for mixed fermions are, $\left|Y_{\mathbf{k}}^{F}\right|=\frac{\left(\Omega_{k, 1}+m_{1}\right)-\left(\Omega_{k, 2}+m_{2}\right)}{2 \sqrt{\Omega_{k, 1} \Omega_{k, 2}\left(\Omega_{k, 1}+m_{1}\right)\left(\Omega_{k, 2}+m_{2}\right)}}|\mathbf{k}|$ and $\Sigma_{\mathbf{k}}^{F}=\sqrt{1-\left|Y_{\mathbf{k}}^{F}\right|^{2}}$, where $\Omega_{k, i}$ are the energies of the free fields.

In the following we also use the property of the mixing generator, $J^{-1}(\xi, t)=J^{+}(\xi, t)=J(-\xi, t)$, to write

$$
{ }_{\lambda}\left\langle 0(\xi, t)\left|: T_{\mu v}^{\lambda}(x):\right| 0(\xi, t)\right\rangle_{\lambda}={ }_{\lambda}\left\langle 0\left|J_{\lambda}^{-1}(-\xi, t): T_{\mu \nu}^{\lambda}(x): J_{\lambda}(-\xi, t)\right| 0\right\rangle_{\lambda},
$$

and we denote with $\Theta(-\xi, x)=J^{-1}(-\xi, t) \Theta(x) J(-\xi, t)$ the operators transformed by $J(-\xi, t)$.

\subsection{Boson Mixing}

One can show that the kinematical part of the energy momentum tensor of free scalar field, $T_{\mu \nu}^{B}(x)=\partial_{\mu} \phi(x) \partial_{\nu} \phi(x)-\frac{1}{2} g_{\mu \nu}\left[\partial^{\rho} \phi(x) \partial_{\rho} \phi(x)-m^{2} \phi^{2}(x)\right]$, is zero when averaged on $|0(\xi, t)\rangle_{B}$, i.e.,

$$
{ }_{B}\left\langle 0(\xi, t)\left|:\left[\partial_{\mu} \phi(x) \partial_{\nu} \phi(x)-\frac{1}{2} g_{\mu \nu} \partial^{\rho} \phi(x) \partial_{\rho} \phi(x)\right]:\right| 0(\xi, t)\right\rangle_{B}=0 .
$$

Then, the energy density and pressure of the condensate become [40]

$$
\rho_{\text {mix }}^{B}=-p_{\text {mix }}^{B}=\left\langle 0\left|: \sum_{i} m_{i}^{2} \phi_{i}^{2}(-\theta, x):\right| 0\right\rangle .
$$

Then, the state equation is the ones of the cosmological constant, $w_{\text {mix }}^{B}=-1$. The condensate, in such a case, can contribute to dark energy. The explicit form of $\rho_{m i x}^{B}$ is

$$
\rho_{\text {mix }}^{B}=\frac{\Delta m^{2} \sin ^{2} \theta}{8 \pi^{2}} \int_{0}^{K} d k k^{2}\left(\frac{1}{\omega_{k, 1}}-\frac{1}{\omega_{k, 2}}\right),
$$


where $K$ is the cut-off on the momenta and $\Delta m^{2}=\left|m_{2}^{2}-m_{1}^{2}\right|$. Equation (13) is used to derive the contributions due to the mixing of axion-photon and of supersymmetric partners of the neutrinos. One has the results below reported.

- In the case of axion-photon mixing, for magnetic field strength $B \in\left[10^{6}-10^{17}\right] G$, axion mass $m_{a} \simeq 2 \times 10^{-2} \mathrm{eV}, \sin _{a}^{2} \theta \sim 10^{-2}$ and a Planck scale cut-off, $\mathrm{K} \sim 10^{19} \mathrm{GeV}$, one has $\rho_{\text {mix }}^{\text {axion }}=2.3 \times 10^{-47} \mathrm{GeV}^{4}$, which is of the same order of the estimated upper bound on dark energy.

- In the case of superpartners of the neutrinos, for $m_{1}=10^{-3} \mathrm{eV}, m_{2}=9 \times 10^{-3} \mathrm{eV}, \sin ^{2} \theta=0.3$ and $K=10 \mathrm{eV}$, one obtains $\rho_{\text {mix }}^{B}=7 \times 10^{-47} \mathrm{GeV}^{4}$. Smaller values of the mixing angle lead to values of $\rho_{\text {mix }}^{B} \sim 10^{-47} \mathrm{GeV}^{4}$ also in the case in which the cut-off is $\mathrm{K}=10^{19} \mathrm{GeV}$ [40].

\subsection{Fermion Mixing}

The kinematical terms of the energy density and pressure of fermion mixed fields are zero when averaged on $|0(\xi, t)\rangle_{B}$, then one has [40]

$$
\begin{aligned}
& \rho_{\text {mix }}^{F}=-\left\langle 0\left|: \sum_{i}\left[m_{i} \psi_{i}^{\dagger}(-\theta, x) \gamma_{0} \psi_{i}(-\theta, x)\right]:\right| 0\right\rangle, \\
& p_{\text {mix }}^{F}=0,
\end{aligned}
$$

where $\psi_{i}(-\theta, x)$ are the flavor fields. The state equation is the one of dark matter, $w_{\text {mix }}^{F}=0$. The explicit form of Equation (14) is

$$
\left.\rho_{m i x}^{F}=\frac{\Delta m \sin ^{2} \theta}{2 \pi^{2}} \int_{0}^{K} d k k^{2}\left(\frac{m_{2}}{\omega_{k, 2}}-\frac{m_{1}}{\omega_{k, 1}}\right)\right] .
$$

For $m_{i} \sim 10^{-3} \mathrm{eV}$ and $K=m_{1}+m_{2}$, one has $\rho_{\text {mix }}^{F}=4 \times 10^{-47} \mathrm{GeV}^{4}$. For $K$ of order of the Plank scale, one has $\rho_{\text {mix }}^{F} \sim \times 10^{-46} \mathrm{GeV}^{4}$. Notice that the quark confinement inside the hadrons should inhibit the gravitational interaction of the quark vacuum condensate. Thus the quark condensate should not affect dark matter. In a similar way, the condensates of mixed bosons, such as kaons, $B^{0}$ and $D^{0}$ mesons should not contribute to dark energy of the universe.

\section{Conclusions}

The vacuum condensates characterizing many systems can contribute to the dark matter and to the dark energy. Dark matter contributions derive by thermal vacuum of intercluster medium, by the vacuum of fields in curved space and by the neutrino flavor vacuum. Dark energy contributions can be given by the axion-photon mixing and by the mixing of supersymmetric partners of neutrino. The formalism presented in the present paper, when applied in a supersymmetric framework, shows that the non-zero energy of vacuum condensate also produces a spontaneous supersymmetry breaking [65-70].

Acknowledgments: Partial financial support from MIUR and INFN is acknowledged.

Conflicts of Interest: The authors declare no conflict of interest.

\section{Reference}

1. De Bernardis, P.; Ade, P.A.R.; Bock, J.J.; Bond, J.R.; Borrill, J.; Boscaleri, A.; Coble, K.; Crill, B.P.; De Gasperis, G.; Farese, P.C.; et al. A flat Universe from high-resolution maps of the cosmic microwave background radiation. Nature 2000, 404, 955-959.

2. Spergel, D.N.; Verde, L.; Peiris, H.V.; Komatsu, E.; Nolta, M.R.; Bennett, C.L.; Halpern, M.; Hinshaw, G.; Jarosik, N.; Kogut, A.; et al. First Year Wilkinson Microwave Anisotropy Probe (WMAP) Observations: Determination of Cosmological Parameters. Astrophys. J. Suppl. 2003, 148, 175-194. 
3. Dodelson, S.; Narayanan, V.K.; Tegmark, M.; Scranton, R.; Budavári, T.; Connolly, A.; Csabai, I.; Eisenstein, D.; Frieman, J.A.; Gunn, J.E.; et al. The Three-dimensional Power Spectrum from Angular Clustering of Galaxies in Early Sloan Digital Sky Survey Data. Astrophys. J. 2002, 572, 140-156.

4. Szalay, A.S.; Jain, B.; Matsubara, T.; Scranton, R.; Vogeley, M.S.; Connolly, A.; Dodelson, S.; Eisenstein, D.; Frieman, J.A.; Gunn, J.E.; et al. Karhunen-Loève Estimation of the Power Spectrum Parameters from the Angular Distribution of Galaxies in Early Sloan Digital Sky Survey Data. Astrophys. J. 2003, 591, 1-11.

5. Riess, A.G.; Strolger, L.G.; Tonry, J.; Casertano, S.; Ferguson, H.C.; Mobasher, B.; Challis, P.; Filippenko, A.V.; Jha, S.; Li, W.; et al. Type Ia Supernovae discoveries at $z>1$ from the Hubble Space Telescope: Evidence for past deceleration and constrains on dark energy evolution. Astrophys. J. 2004, 607, 665-687.

6. Amendola, L.; Tsujikawa, S. Dark Energy: Theory and Observations; Cambridge University Press: Cambridge, UK, 2010.

7. Nojiri, S.; Odintsov, S.D.; Oikonomou, V.K. Modified Gravity Theories on a Nutshell: Inflation, Bounce and Late-time Evolution. Phys. Rep. 2017, 692, 1-104.

8. Rubin, V. The rotation of spiral galaxies. Science 1983, 220, 1339-1344.

9. Nojiri, S.; Odintsov, S.D. Unified cosmic history in modified gravity: From $F(R)$ theory to Lorentz non-invariant models. Phys. Rep. 2011, 505, 59-144.

10. Silvestri, A.; Trodden, M. Approaches to understanding cosmic acceleration. Rep. Prog. Phys. 2009, 72, 096901.

11. Frieman, J.A.; Turner, M.S.; Huterer, D. Dark energy and accelerating Universe. Annu. Rev. Astron. Astrophys. 2008, 46, 385-432.

12. Durrer, R.; Maartens, R. Dark energy and dark gravity: Theory overview. Gen. Relativ. Gravit. 2008, 40, 301-328.

13. Sami, M. Models of Dark Energy. Lect. Notes Phys. 2007, 720, 219-256.

14. Copeland, E.J.; Sami, M.; Tsujikawa, S. Dynamics of dark energy. Int. J. Mod. Phys. D 2006, 15, 1753-1936.

15. Capozziello, S. Curvature quintessence. Int. J. Mod. Phys. D 2002, 11, 483-492.

16. Capozziello, S.; Lambiase, G. Higher-Order Corrections to the Effective Gravitational Action from Noether Symmetry Approach. Gen. Relativ. Gravit. 2000, 32, 295-311.

17. Lambiase, G.; Mohanty, S.; Prasanna, A.R. Neutrino coupling to cosmological background: A review on gravitational Baryo/Leptogenesis. Int. J. Mod. Phys. D 2013, 22, 1330030.

18. Sotiriou, T.P.; Faraoni, V. $f(R)$ theories of gravity. Rev. Mod. Phys. 2010, 82, 451-497.

19. De Felice, A.; Tsujikawa, S. $f(R)$ theories. Living Rev. Relativ. 2010, 13, 3.

20. Schützhold, R. Small Cosmological Constant from the QCD Trace Anomaly? Phys. Rev. Lett. 2002, 89, 081302.

21. Thomas, E.C.; Urban, F.R.; Zhitnitsky, A.R. The cosmological constant as a manifestation of the conformal anomaly? J. High Energy Phys. 2009, 2009, 43.

22. Klinkhamer, F.R.; Volovik, G.E. Vacuum energy density kicked by the electroweak crossover. Phys. Rev. D 2009, 79, 063527.

23. Urban, F. R.; Zhitnitsky, A.R. The cosmological constant from the ghost. A toy model. Phys. Rev. D 2009, 80, 063001.

24. Klinkhamer, F.R.; Volovik, G.E. Gluon condensate, modified gravity, and the accelerating Universe. Phys. Rev. D 2009, 80, 083001.

25. Alexander, S.; Biswas, T.; Calcagni, G. Cosmological Bardeen-Cooper-Schrieffer condensate as dark energy. Phys. Rev. D 2010, 81, 043511.

26. Popławski, N.J. Cosmological constant from quarks and torsion. Ann. Phys. 2011, 523, 291.

27. Bertone, G.; Hooper, D.; Silk, J. Particle dark matter: Evidence, candidates and constraints. Phys. Rep. 2005, 405, 279-390.

28. De Martino, I. $f(R)$-gravity model of the Sunyaev-Zeldovich profile of the Coma cluster compatible with Planck data. Phys. Rev. D 2016, 93, 124043.

29. De Laurentis, M.; De Martino, I.; Capozziello, S. Constraining $f(R)$ gravity by the Large Scale Structure. Universe 2015, 1, 123-157.

30. De Laurentis, M.; De Martino, I.; Atrio-Barandela, F.; Capozziello, S. Constraining $f(R)$ gravity with Planck data on galaxy cluster profiles. Mon. Not. R. Astron. Soc. 2014, 442, 921-928.

31. De Laurentis, M.; De Martino, I. Probing the physical and mathematical structure of $f(R)$ gravity by PSR J0348 + 0432. Int. J. Geom. Methods Mod. Phys. 2015, 12, 1550040.

32. De Laurentis, M.; De Martino, I. Testing $f(R)$-theories using the first time derivative of the orbital period of the binarypulsars. Mon. Not. R. Astron. Soc. 2013, 431, 741-748. 
33. Frigerio Martins, C.; Salucci, P. Analysis of rotation curves in the framework of R n gravity. Mon. Not. Roy. Astron. Soc. 2007, 381, 1103-1108.

34. D'Amico, G.; Kamionkowski, M.; Sigurdson, K. Dark Matter Astrophysics. arXiv 2007, arXiv:0907.1912.

35. Bertone, G. (Ed.) Particle Dark Matter: Observations, Models and Searches; Cambridge University Press: Cambridge, UK, 2010.

36. Bottino, A.; Fornengo, N. Dark matter and its particle candidates. In Non-Accelerator Particle Physics; Taylor \& Francis: Oxford, UK, 1988.

37. Ferraro, S.; Schmidt, F.; Hu, W. Cluster Abundance in $f(R)$ Gravity Models. Phys. Rev. D 2011, 83, 063503.

38. Lombriser, L.; Schmidt, F.; Baldauf, T.; Mandelbaum, R.; Seljak, U.; Smith, R.E. Cluster Density Profiles as a Test of Modified Gravity. Phys. Rev. D 2012, 85, 102001.

39. Schmidt, F.; Vikhlinin, A.; Hu, W. Cluster constraints on $f(R)$ gravity. Phys. Rev. D 2009, 80, 083505.

40. Capolupo, A. Dark matter and dark energy induced by condensates. Adv. High Energy Phys. 2016, 2016, 8089142.

41. Hawking, S.W. Particle Creation by Black Holes. Commun. Math. Phys. 1975, 43, 199-220.

42. Unruh, W.G. Notes on black hole evaporation. Phys. Rev. D 1976, 14, 870.

43. Schwinger, J.S. On gauge invariance and vacuum polarization. Phys. Rev. 1951, 82, 664.

44. Bardeen, J.; Cooper, L.N.; Schrieffer, J.R. Theory of superconductivity. Phys. Rev. 1957, 108, 1175.

45. Iorio, A. Weyl-Gauge Symmetry of Graphene. Ann. Phys. 2011, 326, 1334-1353.

46. Takahasi, Y.; Umezawa, H. Thermo field dynamics. Collect. Phenom. 1975, 2, 55.

47. Umezawa, H.; Matsumoto, H.; Tachiki, M. Thermo Field Dynamics and Condensed States; North-Holland Publishing Company: Amsterdam, The Netherlands, 1982.

48. Umezawa, H. Advanced Field Theory: Micro, Macro, and Thermal Physics; American Institute of Physics: New York, NY, USA, 1993; p. 238.

49. Casimir, H.B.G.; Polder, D. The Influence of Retardation on the London-van der Waals Forces. Phys. Rev. 1948, 73, 360-372.

50. Casimir, H.B.G. On the attraction between two perfectly conducting plates. Proc. Kon. Ned. Akad. Wetensch. 1948, 51, 793-795.

51. Birrell, N.D.; Davies, P.C.W. Quantum Fields in Curved Space. Cambridge University Press: Cambridge, UK, 1984.

52. Blasone, M.; Capolupo, A.; Vitiello, G. Quantum field theory of three flavor neutrino mixing and oscillations with CP violation. Phys. Rev. D 2002, 66, 025033.

53. Blasone, M.; Capolupo, A.; Romei, O.; Vitiello, G. Quantum field theory of boson mixing. Phys. Rev. D 2001, $63,125015$.

54. Capolupo, A.; Ji, C.-R.; Mishchenko, Y.; Vitiello, G. Phenomenology of flavor oscillations with non-perturbative effects from quantum field theory. Phys. Lett. B 2004, 594, 135-140.

55. Albareti, F.D.; Cembranos, J.A.R.; Maroto, A.L. Vacuum energy as dark matter. Phys. Rev. D 2014, 90, 123509.

56. Capolupo, A.; Lambiase, G.; Vitiello, G. Thermal Condensate Structure and Cosmological Energy Density of the Universe. Adv. High Energy Phys. 2016, 2016, 3127597.

57. Parker, L.; Fulling, S.A. Adiabatic regularization of the energy-momentum tensor of a quantized field in homogeneous spaces. Phys. Rev. D 1974, 9, 341.

58. Fulling, S.A.; Parker, L. Renormalization in the theory of a quantized scalar field interacting with a Robertson-Walker space-time. Ann. Phys. 1974, 87, 176-204.

59. Capolupo, A.; Capozziello, S.; Vitiello, G. Neutrino mixing as a source of dark energy. Phys. Lett. A 2007, 363, 53-56.

60. Capolupo, A.; Capozziello, S.; Vitiello, G. Dark energy and particle mixing. Phys. Lett. A 2009, 373, 601-610.

61. Capolupo, A.; Capozziello, S.; Vitiello, G. Dark energy, cosmological constant and neutrino mixing. Int. J. Mod. Phys. A 2008, 23, 4979-4990.

62. Blasone, M.; Capolupo, A.; Capozziello, S.; Vitiello, G. Neutrino mixing, flavor states and dark energy. Nucl. Instrum. Methods A 2008, 588, 272-275.

63. Blasone, M.; Capolupo, A.; Vitiello, G. Particle mixing, flavor condensate and dark energy. Prog. Part. Nucl. Phys. 2010, 64, 451-453.

64. Blasone, M.; Capolupo, A.; Capozziello, S.; Carloni, S. Neutrino mixing contribution to the cosmological constant. Phys. Lett. A 2004, 323, 182-189. 
65. Capolupo, A.; Di Mauro, M.; Iorio, A. Mixing-induced Spontaneous Supersymmetry Breaking. Phys. Lett. A 2011, 375, 3415-3418.

66. Capolupo, A.; Di Mauro, M. Spontaneous Supersymmetry Breaking Induced by Vacuum Condensates. Phys. Lett. A 2012, 376, 2830-2833.

67. Capolupo, A.; Di Mauro, M. Vacuum condensates, flavor mixing and spontaneous supersymmetry breaking. Acta Phys. Polon. B 2013, 44, 81.

68. Capolupo, A.; Vitiello, G. Spontaneous supersymmetry breaking probed by geometric invariants. Adv. High Energy Phys. 2013, 2013, 850395.

69. Capolupo, A.; Di Mauro, M. Vacuum condensates as a mechanism of spontaneous supersymmetry breaking. Adv. High Energy Phys. 2015, 2015, 929362.

70. Capolupo, A. Quantum vacuum, dark matter, dark energy and spontaneous supersymmetry breaking. arXiv 2017, arXiv:1708.08769.

(C) 2017 by the authors. Licensee MDPI, Basel, Switzerland. This article is an open access article distributed under the terms and conditions of the Creative Commons Attribution (CC BY) license (http:/ / creativecommons.org/licenses/by/4.0/). 